\title{
NUEVA ESPECIE FÓSIL DE CERION RÖDING, 1798 \\ (MOLLUSCA: PULMONATA: CERIONIDAE) DE CUBA ORIENTAL
}

\author{
Alexis Suárez Torres \\ Sociedad Cubana de Zoología, 35 No 2627 e/ 26A y Final. Sta. María del Rosario. CP 19330, \\ La Habana, Cuba. alexys02@nauta.cu
}

\section{RESUMEN}

Se describe en estado fósil el taxon Cerion petreus sp. nov. con localidad tipo en El Guanal, Punta de Maisí, provincia de Guantánamo. Este hallazgo permite ubicar la existencia del género en el oriente de Cuba desde Plioceno-Pleistoceno inferior.

Palabras clave: Mollusca, Pulmonata, Cerionidae, Cerion petreus sp. nov., Punta de Maisí, Cuba.

Title: New fossil species of Cerion Röding, 1798 (Mollusca: Pulmonata: Cerionidae) from Eastern Cuba.

\section{ABSTRACT}

Cerion petreus sp. nov. is described on fossil state, with type locality at El Guanal, Punta de Maisí, Guantánamo province. This finding allows us to report the occurrence of this genus in Eastern Cuba since Pliocene to Lower Pleistocene.

Keywords: Mollusca, Pulmonata, Cerionidae, Cerion petreus, sp. nov., Punta de Maisí, Cuba.

\section{INTRODUCCIÓN}

De aproximadamente 600 especies y subespecies descritas para el género de moluscos terrestres Cerion Röding, 1798, hay para la malacofauna cubana 147 formas descritas según Espinosa y Ortea (2009), las que hasta el momento han sido reportadas para localidades costeras. En la totalidad del género, desde el Cretácico Superior hasta el Pleistoceno/ Holoceno han sido descritos 57 táxones fósiles según Harasewych (2012), aunque solo reconoce una especie para Cuba (Cerion barroi Aguayo y Jaume, 1957), siendo citada en la literatura Cerion dickersoni Richards, 1935, en estado fósil por los autores Jaume (1975) y Espinosa y Ortea (1999).

La existencia de conchas fosilizadas de moluscos de la familia Cerionidae Pilsbry, 1901, en terreno pleistocénico de la región norte-occidental de la isla de Cuba, ha sido reportada para las localidades de Miramar, en La Habana ( $C$. barroi) y Monserrate, en Matanzas $(C$. dickersoni). Además, la especie viviente Cerion tridentatum Pilsbry y Vanatta, 1895 se ha reportado en estado fósil para la localidad de Guanabo, en La Habana (Torre y Kojumdgieva, 1985), extendiendo su población en la actualidad desde Barrio Caribe hasta el Rincón de Guanabo (datos del autor).

El reporte fósil de los Ceriónidos en general se extiende desde el Cretácico Superior de Montana (Roth y Hartman, 1998) hasta el Pleistoceno, siendo muy abundantes en los depósitos más jóvenes de las Bahamas (Hearty y Kaufman, 2009). La historia fósil del género para la Florida data para el Oligoceno/ Mioceno temprano (Petuch, 2004). Recientemente, el género fósil Brasilennea, representado por tres especies del Paleoceno de la cuenca del Itaboraí, Brasil, ha sido transferido de la Familia Streptaxidae hacia la familia Cerionidae (Salvador et al., 2011; Salvador y Simone, 2012). 
En el presente trabajo se describe una especie fósil de Cerion que demuestra la presencia y antigüedad del género en la región oriental de Cuba.

\section{OBJETIVO}

- Describir una nueva especie en estado fósil para el género Cerion.

\section{MATERIALES Y MÉTODOS}

Se trabajó con 31 conchas fosilizadas de la familia Cerionidae recolectadas durante expedición en fecha 23-25 de septiembre del 2013. La colecta se hizo en la localidad El Guanal, Maisí, la zona más oriental de la provincia Guantánamo. Las conchas fueron comparadas con especímenes de colecciones depositadas en el Instituto de Ecología y Sistemática de Cuba. Además, se utilizó la información disponible en el sitio Cerion website [http://invertebrates. si.edu/cerion/] (Harasewych, 2009). Las especies actuales Cerion paucicostatum paucicostatum Torre, 1929; Cerion orientale Clench y Aguayo, 1951; Cerion caroli aedili Aguayo y Torre, 1951; Cerion alberti Clench y Aguayo, 1949; y Cerion dimidiatum Pfeiffer, 1847, fueron usadas para definir la validez de este táxon, ya que comparten mayor cantidad de caracteres conquiológicos, también la nueva especie fue comparada con C. barroi Aguayo y Jaume, 1957 y $C$. dickersoni Richards, 1935, únicos fósiles descritos dentro del género para Cuba hasta la actualidad, haciéndose uso de las descripciones originales, así como con la especie actual, todas encontradas en yacimientos pleistocénicos.

Para análisis estadístico y selección de la serie tipo, se utilizó material proveniente de la colección del autor, el que fue depositado en la Colección Paleontológica de las Colecciones Zoológicas del Instituto de Ecología y Sistemática.

Todas las medidas son dadas en milímetros. Debido al estado de deterioro de la mayoría de los ejemplares, las medidas tomadas para análisis estadístico solo incluyeron el diámetro de la concha en la zona superior de la penúltima vuelta y su amplitud (Fig. 1), para lo cual se utilizó un calibrador digital Powerfix. El procesamiento estadístico se llevó a cabo con el programa: digitalestal StatSoft, Inc. (2003) STATISTICA (data analysis software system), version 6. www. statsoft.com. La descripción de las variables incluye los siguientes valores: Media (M), Límites de confianza al 95\% X \pm Sx: Límite inferior (LI) y Límite superior (LS), Valores mínimos (Mín.), Valores máximos (Máx.) y Desviación estándar.

Luego de determinarse que se trataba de un nuevo taxon, se procedió a la descripción.

Abreviaturas: IES (Instituto de Ecología y Sistemática); mm (milímetros); long (Longitud), diam (Diámetro); m.s.n.m. (metros sobre el nivel del mar); MNHNCu (Museo Nacional de Historia Natural, La Habana, Cuba); SCG (Sociedad Cubana de Geología); A (Alexis Suárez Torres, autor).

\section{TAXONOMÍA}

Clase GASTROPODA, Subclase PULMONATA Orden STYLOMMATOPHORA, Familia Cerionidae Pilsbry, 1901 Género Cerion Röding, 1798, Subgénero Strophiops Dall, 1894 


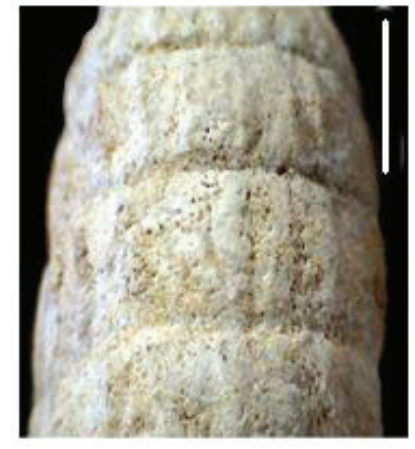

A

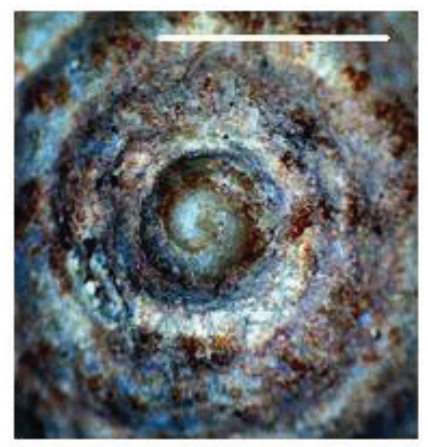

C

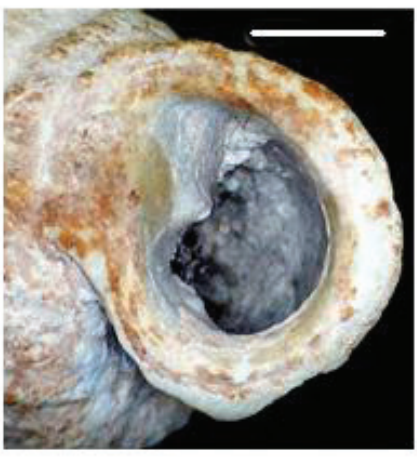

B

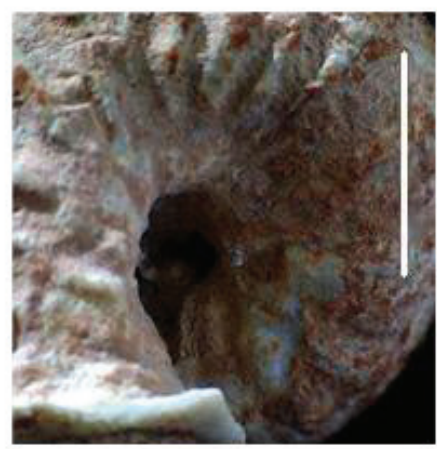

D

Figura 1, A-D. Cerion petreus sp. nov. A, costillas. B, peristoma y diente parietal. C, núcleo, protoconcha y teleoconcha. D, ombligo. Escala: $5 \mathrm{~mm}$.

Descripción. Concha grande, de forma cilíndrica y entre 10 y 10 1/2 vueltas de espira, (holotipo 37.13 de long., 16.90 de diám.). Núcleo corto, menos ancho que la protoconcha, la que muestra los primeros giros más anchos que los giros de inicio de la teleoconcha (Fig. 1C). Región apical poco elevada y ancha. Posteriormente, la concha adquiere forma cilíndrica de lados rectos. Costillas axiales espaciadas, romas, con base ancha, distanciándose progresivamente entre ellas (Fig. 1A). Última vuelta que se expande y sobresale a medida que se acerca al peristoma, en la que se cuentan entre 17 y 19 costillas. Abertura amplia, de forma circular con tendencia a ser oblicua, con peristoma grueso, el que disminuye el grosor hacia el borde, reflejándose filoso ligeramente hacia atrás. Callo parietal del mismo grosor que el peristoma, cerrando los bordes de éste; algunas conchas presentan un doble peristoma. Diente parietal notable, alejado de la abertura (Fig. 1B). Diente columelar más profundo que el parietal y menos elevado. Ombligo amplio y profundo (Fig. 1D). 
Localidad tipo. El Guanal, Punta de Maisí, Guantánamo, en suelo arenoso con conglomerado. En $\operatorname{los} 20^{\circ} 13^{\prime} \mathrm{N} ; 74^{\circ} 08^{\prime} \mathrm{O}$.

Estratigrafía. En terrenos de la formación Jaimanitas, del Pleistoceno Superior, de unos 100 mil a 130 mil años de antigüedad (Iturralde-Vinent, 2011).

Material tipo. Holotipo (Fig. 2) y 30 Paratipos (Figs. 3 y 4) depositados en IES.

Distribución. Se encuentran conchas fosilizadas en la superficie del terreno desde El Guanal (localidad tipo), hasta La Palizada, apareciendo nuevamente en Punta Negra, desde los $20^{\circ} 13^{\prime}$ $\mathrm{N}$; $74^{\circ} 08^{\prime} \mathrm{O}$; hasta los $20^{\circ} 05^{\prime} \mathrm{N}$; $74^{\circ} 14^{\prime} \mathrm{O}$; de Maisí, Guantánamo.

Etimología. Del latín Petreus (Piedra).

Observaciones. El área de distribución de esta población pudo haber abarcado unos $17 \mathrm{~km}$ lineales a lo largo de la costa, según los indicios del hallazgo. La aparición en éste terreno posibilita ubicar la existencia del género en la región oriental de Cuba desde el Plioceno al Pleistoceno inferior.

Los ejemplares se encontraron a una altura aproximada de $1 \frac{1}{2} 2$ m.s.n.m. expuestos sobre la tierra, diseminados sin una posición específica.

En la actualidad, en éstas localidades viven las especies Cerion paucicostatum paucicostatum Torre, 1929, y Cerion orientale Clench y Aguayo, 1951, las que podrían ser formas subsecuentes de Cerion petreus, sp. nov., formadas inicialmente a partir de poblaciones alopátricas, que con el tiempo, al haber desaparecido la forma anterior, se hayan establecido en el nuevo espacio disponible, adaptándose a las condiciones imperantes.

Diagnosis diferencial. La especie Cerion petreus sp. nov. difiere de C. barroi, C. dickersoni, $C$. tridentatum, $C$. paucicostatum paucicostatum y $C$. orientale en su mayor talla (longitud= $40.03 \mathrm{~mm}$; diámetro $=17.36 \mathrm{~mm}$ ) [barroi, dickersoni, paucicostatum, orientale $=$ longitud $<$ 35; diámetro < 13]. De C. barroi; $C$. dickersoni y C. tridentatum difiere además en su ápice poco elevado, costillas axiales imperceptibles y separadas; así como de $C$. paucicostatum paucicostatum se separa por tener menor cantidad de costillas y un diente columelar más profundo. $C$. orientale además de su menor talla se diferencia de $C$. petreus sp. nov. en poseer superficie lisa y forma del cuerpo no cilíndrica. C. caroli aedili comparte el carácter talla grande con el nuevo taxon y difiere del mismo en la superficie totalmente acostillada y forma fusiforme. C. alberti y $C$. dimidiatum poseen conchas de apariencia cuadrada, con lados rectos y ápices planos, diferenciándose fácilmente de la especie nueva.

Tanto el tamaño, como el aspecto general de la concha, hacen de Cerion petreus sp. nov. una especie diferente del resto de los ceriónidos cubanos actuales.

Comentario. Durante el análisis estadístico, no se apreció correlación de las variables consideradas en las muestras $(\mathrm{r}=023 ; \mathrm{p} \geq 0.05)$. La media del diámetro de concha es algo más del doble que el valor del ancho de la penúltima espira; esta misma tendencia proporcional de ambas dimensiones lineales se mantiene cuando se comparan los valores reflejados en los límites de confianza inferior (LI) y superior (LS), así como en los valores mínimos y máximos. Aunque la desviación estándar del diámetro es superior al del ancho de la penúltima vuelta, evidencian baja variabilidad, por lo que las medidas de ambas variables evidenciaron tener relativa estabilidad en esta especie fósil, lo que posibilita utilizarlas como buenos caracteres diagnósticos para la misma (Tabla I). 


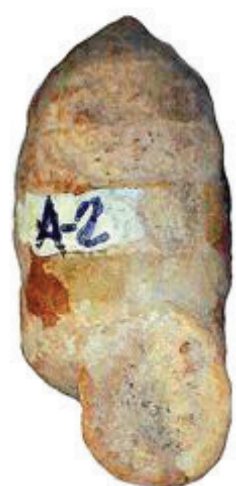

$\mathbf{A}$

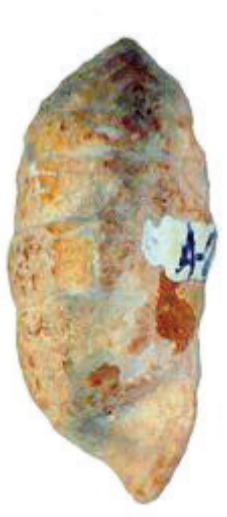

C

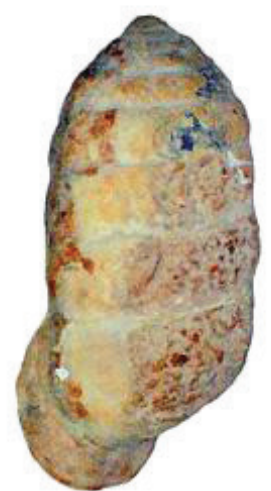

B

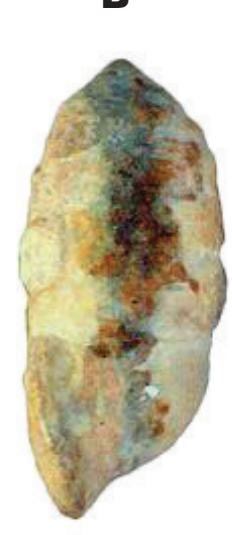

D

\section{A-2}

Figura 2, A-D. Holotipo de Cerion petreus sp. nov. A, vista inferior. B, vista superior. C, vista lateral derecha. D, vista lateral izquierda. Escala: $10 \mathrm{~mm}$.

Tabla I. Descripción estadística de dos variables de Cerion petreus sp. nov.

\begin{tabular}{|c|c|c|c|c|c|c|c|}
\hline Variable & $\mathrm{N}$ & Media & LI -95,000 \% & LS +95,000\% & Mín. & Máx. & $\begin{array}{c}\text { Desviación } \\
\text { estándar }\end{array}$ \\
\hline Diámetro & 39 & 15,7 & 15,3 & 16,1 & 14 & 18,6 & 1,2 \\
\hline Amplitud & 39 & 6,5 & 6,3 & 6,7 & 5,3 & 7,9 & 0,5 \\
\hline
\end{tabular}




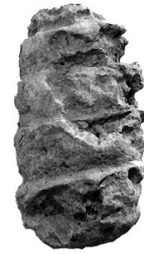

A- 1

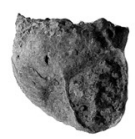

A- 4

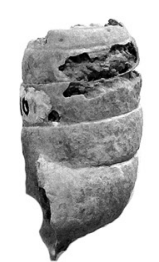

A- 10

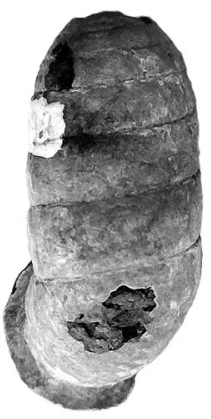

A- 15

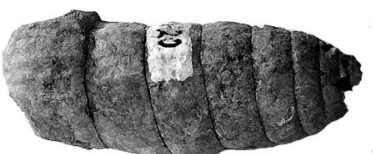

A- 20

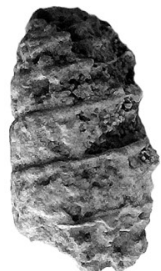

A- 3

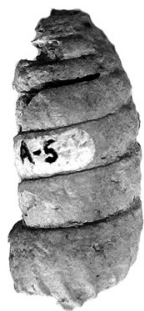

A- 5

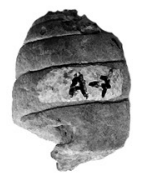

A- 7

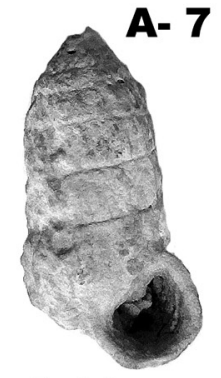

A-11

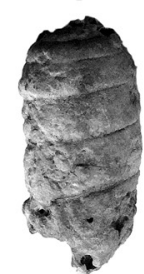

A- 8

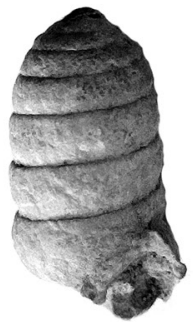

A- 6
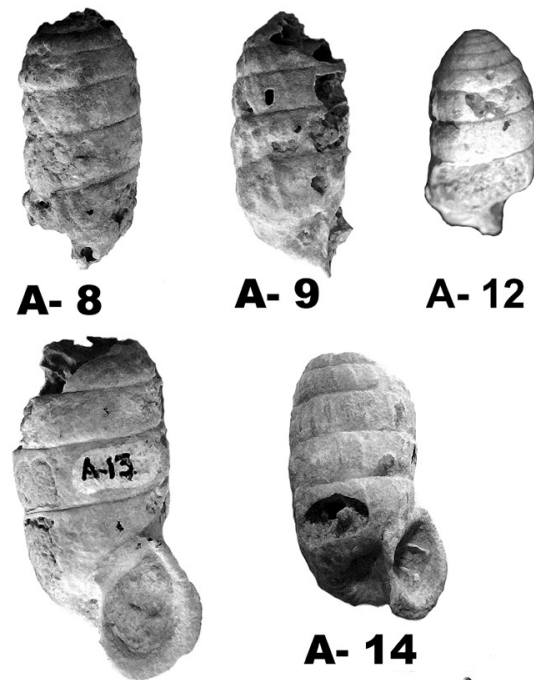

A- 9

A- 12

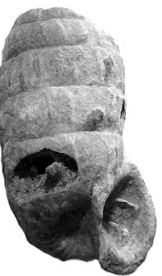

$$
\text { A- } 14
$$

A-13
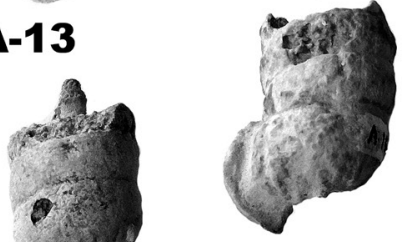

A- 16

A- 17

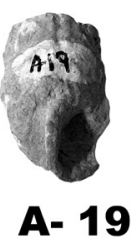

A- 19

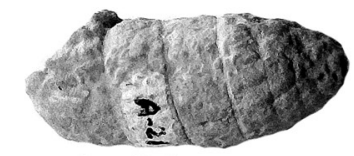

A- 21 

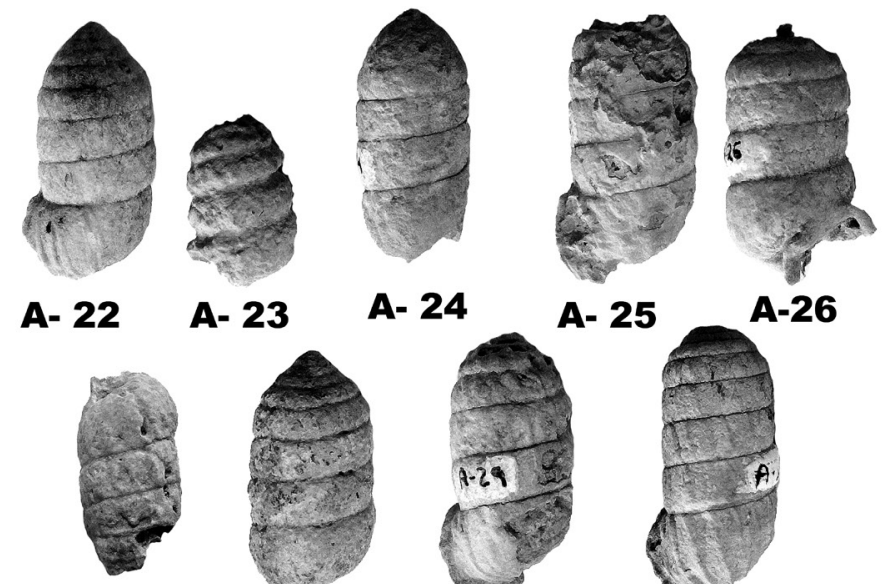

A- 23

A- 24

A- 25

A-26

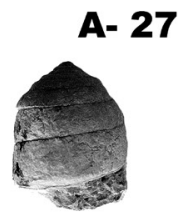

A- 31

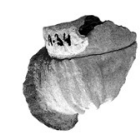

A- 34
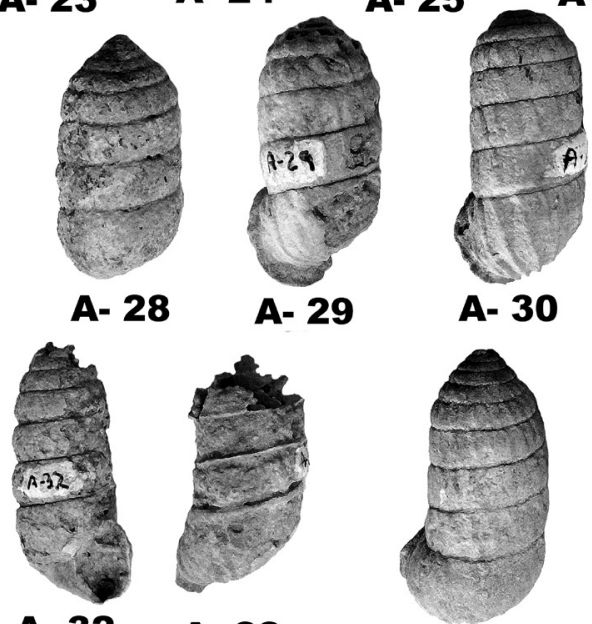

A- 32 A- 33
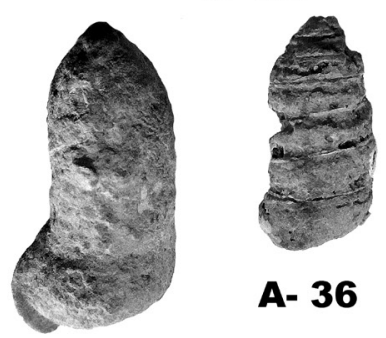

A- 38

A- 36

A- 35

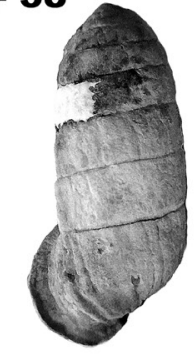

A- 37

Figura 4, A22-A38. Paratipos de Cerion petreus sp. nov. Escala: $7 \mathrm{~mm}$.

\section{AGRADECIMIENTOS}

A IDEA WILD por donar equipamiento que ha sido utilizado en la investigación. A Esteban Gutiérrez (MNHNCu) y Manuel Iturralde (SCG) por la revisión crítica del documento. A José Antonio Ruiz, Guillermo Ponce de León y Roger Rodríguez por su colaboración durante el trabajo de campo y en especial a Alexis Morales, quien me hizo llegar las primeras muestras recolectadas, haciendo de guía posteriormente durante todo el periplo. A Javier Rabaza, de la Empresa Nacional para la protección de la Flora y la Fauna en Baracoa, Guantánamo. A Alexander Cadena y Osmay Samón, de la Filial Universitaria MES, en Guantánamo; Alejandro Fernández, por la ayuda brindada durante el análisis estadístico; Nelsy Blet, por haberme acogido en su hogar durante la estancia en la provincia de Guantánamo; Gladis Gil por su ayuda durante el trabajo de gabinete; Nayla García y Jans Morffe, por las orientaciones pertinentes. 


\section{LITERATURA CITADA}

Espinosa, J. y J. Ortea. 1999. Moluscos terrestres del archipiélago cubano. Avicennia, suplemento 2: $1-137$.

Jaume, M. L. 1975. Catálogo de los moluscos terrestres cubanos del género Cerion (Mollusca: Pulmonata: Ceriidae) (con una bibliografía general). Catalogo de la fauna cubana, 37: 1-47.

Harasewych, M. G. (Ed.). 2009. Cerion v 1.02. Cerion, a web based resource for Cerion research and identification. National Museum of Natural History, Smithsonian Institution. http:// invertebrates.si.edu/Cerion/.

Harasewych, M. G. 2012. The fossil record and phylogeography of the family Cerionidae (Gastropoda: Pulmonata), with the description of a new species from the Pleistocene of Florida. The Nautilus, 126 (4): 119-126.

Hearty, P. J., Kaufman, D. S. 2009. A Cerion-based chronostratigraphy and age model from the central Bahama Islands: Amino acid racemization and $14 \mathrm{C}$ in land snails and sediments. Quaternary Geochronology, 4: 148-159.

Iturralde-Vinent, M. A. (Ed.). 2011. Compendio de Geología de Cuba y del Caribe. Primera Edición. DVD-ROM. Editorial CITMATEL, la Habana, Cuba.

Petuch, E. J. 2004. Cenozoic Seas, the View from Eastern North America. CRC Press, Boca Raton, 308 pp.

Roth, B. and J. H. Hartman. 1998. A probable Cerion (Gastropoda: Pulmonata) from the uppermost Cretaceous Hell Creek Formation, Garfield County, Montana. Paleobios, 18 (2-3): 16-20.

Salvador, R. B., B. Rowson, and L. R. L. Simone. 2011. Rewriting the fossil history of Cerionidae (Gastropoda: Pulmonata): new family assignment of the Brazilian Paleocene genus Brasilennea Maury, 1935. Journal of Molluscan Studies, 77: 445-447.

Salvador, R. B. y L. R. L. de Simone. 2012. New fossil pulmonate snails from the Paleocene of Itaboraí Basin, Brazil (Pulmonata Cerionidae Strophocheilidae, Orthalicidae). Archiv für Molluskenkunde, 141: 43-50.

Torre, A. de la y E. Kojumdgieva. 1985. Asociaciones y niveles faunales de moluscos del Plioceno-Cuaternario del occidente de Cuba, y sus implicaciones estratigráficas. Reporte de Investigación del Instituto de Geología y Paleontología, 5.

[Recibido: 02 de febrero, 2015. Aceptado para publicación: 26 de julio, 2015] 